\title{
Jolanta Sękowska
}

Maria Curie-Skłodowska-Universität, Lublin

DOI: $10.19195 / 0435-5865.141 .20$

\section{Rekursion und syntaktische Komplexität im Lichte der psycho- und neurolinguistischen Forschung}

\begin{abstract}
"In some sense it is like looking at a tapestry: seen from the front, it looks like many little points of different colors, one next to the other, forming a vivid image. But if we look at the back, we discover a complex structure of woven threads that makes almost no visual sense. Individual threads emerge on the surface and then dive, disappearing on this side while creating the image of the tapestry on the other. In a way, syntax resembles a tapestry: if we look at it superficially, it appears as a simple row of words, arranged next to each other in a sound and coherent way. But if we manage to look at it "from behind," we discover the hidden and intricate structure that connects them at a distance." (Moro 2008: 56)
\end{abstract}

\section{Einführung}

Die Veröffentlichung des Artikels von Hauser, Chomsky \& Fitch (2002) entfachte eine lebhafte Diskussion, ob die Rekursion als eine exklusive Eigenschaft der Sprachfähigkeit im engen Sinne (faculty of language - narrow sense, FLN) zu gelten hat, d.h. als die grundlegende Eigenschaft des internen komputationellen Systems, dank derer die FLN diskrete Unbegrenztheit erbringt. ${ }^{1}$ Laut Hauser, Chomsky \& Fitch (2002) und Fitch, Hauser \& Chomsky (2005) bildet die Rekursion den Kern der menschlichen, biologisch determinierten Sprachfähigkeit. Mittlerweile wird aber der nicht-biologische, kulturelle Aspekt der Sprache nicht

${ }^{1}$ Die sprachliche Rekursion ist mit der Diskretheit gekoppelt. Die syntaktischen Strukturen sind diskret, weil der wahrgenommene, kontinuierliche Redestrom in eine Kombination der diskreten Einheiten zersplittert wird. Diese Eigenschaft der Sprachfähigkeit wird diskrete Infinität genannt (Epstein \& Hornstein, (2005); Fitch, Hauser, \& Chomsky, (2005); Pullum \& Scholz, (2010)). 
abgelehnt. Von der FLN ist die Sprachfähigkeit im weiten Sinne (faculty of language - broad sense, FLB) abzugrenzen, die neben der FLN auch sensomotorische und konzeptuell-intentionale Systeme mit einschließt. Während die FLN den Kern der menschlichen Sprachfähigkeit ausmacht, vermuten Hauser, Chomsky \& Fitch (2002), dass die Eigenschaften der sensomotorischen und konzeptuell-intentionalen Systeme, welche die Sprachlaute wahrnehmen und produzieren sowie Bedeutungsstrukturen verarbeiten lassen, mit anderen Lebewesen geteilt werden, wobei die Differenzen zwischen den Menschen und beispielsweise nicht-menschlichen Primaten in dieser Hinsicht eher quantitativer als qualitativer Natur sind.

Die ersten Argumente gegen die These von der Rekursion als definierender Eigenschaft der Sprache kommen aus den empirischen Untersuchungen zur Universalitätsthese. Everett (2005) argumentiert, dass Pirahã, die einzige heute noch gesprochene Sprache der Mura-Sprachfamilie, keine rekursiven Strukturen aufweist. Seit der Publikation von Everetts Aufsatz (Everett (2005) gibt es eine fortwährende Diskussion zu diesem Thema (Everett (2007a, b); Sakel (2007); Slobin (2007); Nevins, Pesetsky, \& Rodrigues (2009); Sakel \& Stapert (2010); Zwart $(2011))^{2}$

Die Ansicht, die Rekursion sei keine ausschließlich sprachspezifische Eigenschaft, wird auch von Jackendoff \& Pinker (2005) und Pinker \& Jackendoff (2005) vertreten. Die Forscher argumentieren, dass die Verarbeitung der rekursiven Strukturen auch im visuellen System stattfindet, und wenden sich damit gegen den Standpunkt von Hauser, Chomsky \& Fitch (2002) und Fitch, Hauser \& Chomsky (2005). Mit der Annahme der Rekursion als Kernaspekt der menschlichen Sprachfähigkeit werden ihrer Meinung nach auch diejenigen Aspekte der Grammatik ignoriert, die zwar nicht rekursiv, aber sprachspezifisch sind (z. B. Kasus, Kongruenz). Darüber hinaus betonen Jackendoff \& Pinker (2005), dass die Systeme der Sprachrezeption und - produktion auch als Teile von der FLN aufgefasst werden sollten.

Anzumerken ist aber, dass die Erforschung der Fähigkeit zur Verarbeitung der rekursiven Strukturen durch mehrere distinkte Auffassungen der Rekursion erschwert ist. Beispielsweise definiert Boeckx (2010: 32) die Rekursion als ,the capacity to embed one thought inside another", während Yang (2006) die Rekursion mit der Selbstreproduktion gleichsetzt:

Grammar works very much the same way: small phrases can be embedded within big ones. Language therefore has the ability of self-reproduction, or recursion, to use a term from mathematics: a phrase may beget another phrase, then another, then yet another. (Hervorhebung im Original; Yang (2006: 103)

${ }^{2} \mathrm{Zu}$ beachten ist dabei, dass Everetts frühere Arbeit (1986) eine umfangreiche Beschreibung der eingebetteten Sätze in Pirahã enthält, während er das Bestehen dieser Strukturen in seiner rezenteren Arbeit (Everett 2005) verneint. Wegen dieser Diskrepanz haben die Forscher, die die Hypothese befürworten, wonach Pirahã rekursive Syntax zulässt, die neuere Analyse verworfen (Zwart 2011). Eine vorsichtigere Untersuchung der Tatsachen über Pirahã-Syntax widerspricht den Ansprüchen Everetts und verweist auf die Anwesenheit der rekursiven Strukturen in der Pirahã-Sprache (Nevins, Pesetsky \& Rodrigues 2009). 
Für Marcus (2006) ist jede Sprache, die $\mathrm{A}^{\mathrm{n}} \mathrm{B}^{\mathrm{n}}$-Strukturen enthält, rekursiv:

The $A^{n} B^{n}$ language (...) is generally assumed to be recursive because new sentences can be formed by successive insertion into the frame $\mathrm{AXB}$, for example $\mathrm{AB}, \mathrm{AABB}, \mathrm{AAABBB}$ and so on. (Marcus 2006: 1117)

$\mathrm{Zu}$ unterscheiden ist aber zwischen der Rekursion auf der Struktur- und der Algorithmusebene Fitch \& Friederici (2012); Martins \& Fitch (2014). Die als rekursiv wahrgenommenen Strukturen können sowohl mit rekursiven als auch nicht-rekursiven Algorithmen generiert werden. Dies bedeutet, dass es nicht leicht festzustellen ist, ob ein System, das rekursive Strukturen produziert, sich eines rekursiven Algorithmus bedient. Im Folgenden wird diese Frage näher behandelt.

\section{Iterative, hierarchische und rekursive Strukturen - definitorische Abgrenzung}

„Iteration" bezieht sich auf das mehrmalige Wiederholen eines Vorgangs. Iterative Prozesse sind weder hierarchisch noch rekursiv; sie dürfen keine hierarchischen Strukturen generieren oder Abhängigkeitsbeziehungen zwischen den Elementen herstellen Fitch (2010); Martins \& Fitch (2014). Demgegenüber beinhalten „hierarchische" Strukturen immer das Einbetten von Elementen innerhalb anderer Elemente, wobei die Einbettung die Gruppierung eines Sets von Elementen in eine höhere Ordnung bedeutet. Erfolgt die hierarchische Einbettung zwischen Bestandteilen derselben Kategorie (z.B. eine Nominalphrase innerhalb einer Nominalphrase), wird sie als rekursiv klassifiziert. Iteration, hierarchische Einbettung und Rekursion dürfen einander nicht gegenübergestellt werden: Die Rekursion umfasst Hierarchie und Iteration. Demzufolge ist es wichtig, die Wissenstypen, die den genannten Prozessen zugrunde liegen, voneinander begrifflich zu trennen.

Die Iteration erfordert das Wissen von der Elementwiederholung. Die hierarchische Einbettung benötigt das Wissen von Abhängigkeitsbeziehungen zwischen einzelnen Komponenten. Die rekursive Einbettung setzt dagegen das Wissen voraus, dass die aufeinander folgenden hierarchischen Abhängigkeiten mit der gleichen Regel generiert werden. Erst dann besteht die Möglichkeit, eine neue hierarchische Ebene über die zuvor spezifizierte zu stellen und zugleich die Konsistenz mit vorhandenen Ebenen auf einem höheren Abstraktionsniveau zu erhalten. Die Rekursion ist somit komplexer als eine bloße Erhöhung der Länge einer Zeichenkette durch Hinzufügung der Wörter an der rechten oder linken Satzseite. Die Wörter können gruppiert werden und bilden Phrasen, die eine interne asymmetrische Struktur haben. Die Asymmetrie resultiert daraus, dass der Kopf einer Phrase und das, was danach folgt, mit einem pronominalen Element ersetzt werden darf, der Kopf selbst und das, was früher erscheint, jedoch nicht. Eine grundlegende Entdeckung ist dabei, dass alle Phrasen eine sehr ähnliche asymmetrische 
Struktur aufweisen und dass ein komplexer Satz eine Phrasenstruktur der gleichen Art hat wie die Phrasen, aus denen er gebildet wird (Chomsky 1986).

\section{Die Verarbeitung der linearen und hierarchischen Strukturen bei Menschen und nicht-menschlichen Primaten}

Die Debatte um die Rekursion gab wichtige Impulse zu zahlreichen empirischen Untersuchungen zur Frage, ob sich die Menschen und nicht-menschlichen Primaten in ihrer Fähigkeit unterscheiden, die rekursiven und nicht-rekursiven iterativ-linearen Strukturen zu verarbeiten, sowie ob die Verarbeitung dieser Strukturen bei Menschen distinkte neuronale Mechanismen involviert. Beide Strukturtypen basieren auf zwei Regeltypen, die durch zwei Grammatiken zugelassen sind: endliche Zustandsgrammatik (finite state grammar, FSG) und Phrasenstrukturgrammatik (phrase structure grammar, PSG). In einer Hierarchie der formalen Grammatiken von Chomsky (1956), der die Grammatiken je nach ihrer generativen Kraft systematisiert hat, nimmt die FSG eine niedrigere Position als die PSG ein. Die FSG kann durch Übergangswahrscheinlichkeit zwischen einer begrenzten Anzahl von Zuständen (z. B. adjazenten Wörtern) völlig spezifiziert werden, ist jedoch nicht genug leistungsfähig, Strukturen der natürlichen Sprache zu generieren. Zwar beinhalten die Sätze der natürlichen Sprache oft Zwischenphrasen oder Zwischenkonstituenten, die aus Wörtern einer spezifischen Kategorie bestehen, zu bemerken ist aber, dass einige Wortkategorien unabhängiger als andere sind, z.B. ist im Deutschen die Übergangswahrscheinlichkeit zwischen einem Artikel und einem Nomen sehr hoch, aber auf ein Nomen kann eine Vielzahl anderer Wörter folgen (Auxiliare, Hauptverben). Eine enge Verbindung der Wortkategorien innerhalb einer Phrase veranschaulicht die Tatsache, dass eine Phrase durch ein einfaches Element ersetzt werden darf (z. B. eine Nominalphrase durch ein Pronomen) und dass nur Phrasen bewegt werden dürfen. Die Relationen zwischen Phrasen werden dagegen durch Abhängigkeiten zwischen Konstituenten selbst bestimmt.

Im Experiment von Fitch \& Hauser (2004) wurden Menschen und nicht-menschliche Primaten (Lisztaffen; sanguinus oedipus) mit FSG- und PSG-Strukturen konfrontiert. Die linear-sequenziellen FSG-Strukturen folgten der Regel $(\mathrm{AB})^{\mathrm{n}}(\mathrm{Abb}$. 1a). In diesem Fall wurden einfache adjazente Sequenzen von zwei Kategorien (A und B) gebildet (z.B. n = 3; ABABAB). Die hierarchischen Strukturen wurden mit der Regel $A^{n} B^{n}$ erzeugt. Eine derartige Regel erlaubt die Generierung von Zentraleinbettungen (z.B. $n=3$; AAABBB) (Abb. 1 b). 
a.

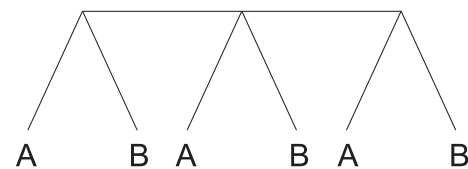

b.

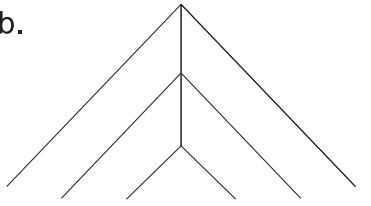

A A A B B B

Abb. 1 Die linear-sequenziellen (AB) $)^{\mathrm{n}}$-Strukturen (a) und die hierarchischen $A^{\mathrm{n}} B^{\mathrm{n}}$ -Strukturen (b)

Die Studie ergab, dass - trotz der Fähigkeit, sequenzielle Regularitäten in den Lautketten zu erkennen - die Lisztaffen nicht imstande sind, einfache $\mathrm{A}^{\mathrm{n}} \mathrm{B}^{\mathrm{n}}$-Strukturen zu verarbeiten, in denen Komponenten in einem Teil der Lautkette mit Komponenten in einem anderen Teil der Lautkette in einer Abhängigkeitsrelation stehen. Die Menschen können aber beide Grammatiktypen leicht erlernen. Fitch \& Hauser (2004) interpretierten ihre Befunde als Evidenz dafür, dass die Verarbeitung der Phrasenstrukturen für die Sprachfähigkeit kritisch ist. Die Menschen, die im Experiment von Fitch \& Hauser (2004) mit lokal-sequenziellen oder mit hierarchischen Strukturen konfrontiert wurden, lehnten die Verletzungen der Regel als „different“ ab und akzeptierten den konsistenten Input als „gleich“. Die Affen orientierten sich signifikant häufiger an Verletzungen. Bei $\mathrm{A}^{\mathrm{n}} \mathrm{B}^{\mathrm{n}}$-Strukturen konnten sie zwischen konsistenten und nicht-konsistenten Inputketten nicht unterscheiden und reagierten auf beide Inputtypen ähnlich.

An dieser Stelle stellt sich die Frage, was genau die Beherrschung von $\mathrm{A}^{\mathrm{n}} \mathrm{B}^{\mathrm{n}}$-Grammatiken durch Menschen oder irgendeine andere Spezies anzeigt. Fitch \& Friederici (2012: 1941) besprechen drei Möglichkeiten der Implementierung von $\mathrm{A}^{\mathrm{n}} \mathrm{B}^{\mathrm{n}}$-Strukturen durch ein Verarbeitungssystem. Die einfachste Strategie ist ,aufzählen und vergleichen“ (Abb. 2a). Eine alternative Strategie erbringt die „zentral-eingebettete“ Struktur und diese ist auch unkompliziert, weil jedes „A“ mit dem vor kurzem gehörten/gesehenen „B“ zusammengebracht wird (Abb. 2b). Eine dritte Strategie ergibt eine asymmetrisch eingebettete Abhängigkeit und erfordert mindestens eine kontextsensitive Grammatik (Abb. 2c). Fitch \& Friederici (2012) verweisen auf die asymmetrische Struktur der zentral-eingebetteten Abhängigkeiten in der natürlichen Sprache (in Bezug auf die Relation zwischen dem Subjekt und dem Verb), was nur die dritte Verarbeitungsmöglichkeit zulässt. In der natürlichen Sprache muss eine vielschichtige hierarchische Struktur erstellt werden, was für eine einfache $\mathrm{A}^{\mathrm{n}} \mathrm{B}^{\mathrm{n}}$-Struktur nicht erforderlich ist. Aus diesem Grunde bedeuten die Ergebnisse der Untersuchungen mit $\mathrm{A}^{\mathrm{n}} \mathrm{B}^{\mathrm{n}}$-Strukturen nicht notwendigerweise, dass die Tiere die asymmetrischen Einbettungen der natürlichen Sprache wie die Menschen verarbeiten können.

Die Verarbeitung von Zentraleinbettungen, die durch die PSG generiert werden, ist jedoch für das Sprachverstehen entscheidend. Die Tatsache, dass die Men- 


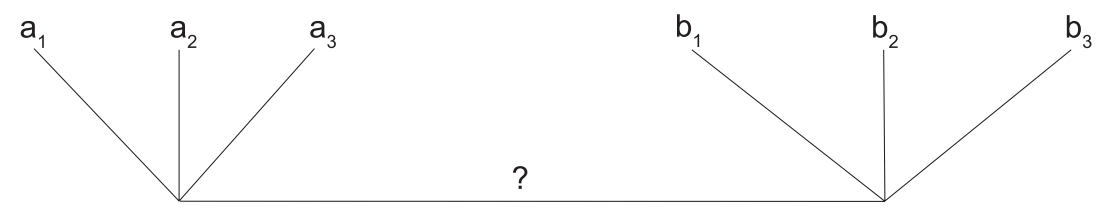

(a)

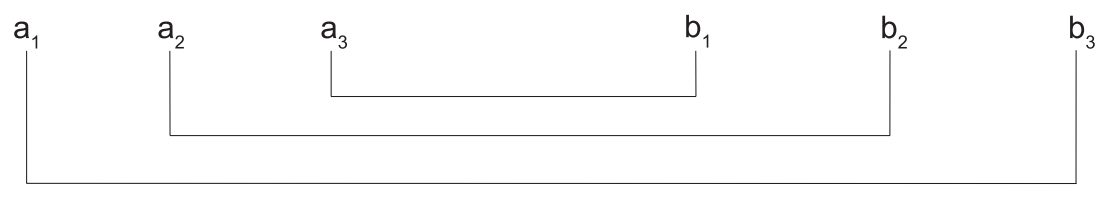

(b)

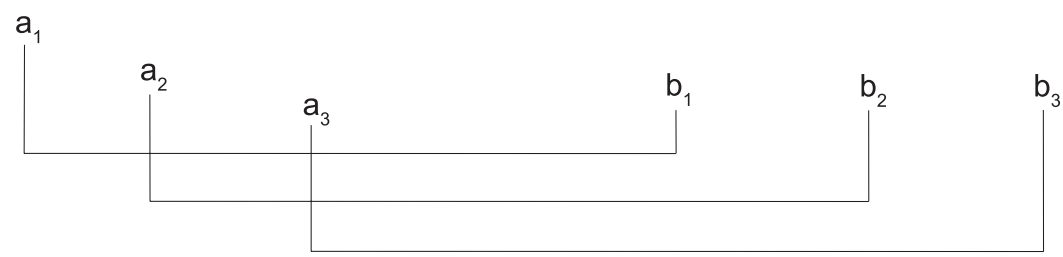

(c)

Abb. 2 Strategien der Verarbeitung von hierarchischen Abhängigkeiten (nach Fitch\&Friederici 2012: 1941)

schen sowohl lokale Sequenzen als auch hierarchische Strukturen verarbeiten und erlernen können, zeugt davon, dass die menschliche Sprachfähigkeit weit über die kognitiven Fähigkeiten der nicht-menschlichen Primaten hinausgeht.

\section{Neuronale Korrelate der FSG- und PSG-Verarbeitung}

Der Frage, wie die komputationellen Anforderungen der FSG und PSG durch das menschliche Gehirn reflektiert werden, wurde in einer Reihe von bildgebenden und neurophysiologischen Studien nachgegangen. Friederici et al. (2006) führten ein fMRI-Experiment durch, um zu prüfen, ob die Verarbeitung der FSG- und PSG-Strukturen durch distinkte Hirnareale unterstützt wird. Den Ausgangspunkt bildeten bisherige Befunde zur Verarbeitung der Sätze mit Objektpermutationen und der lokalen Abhängigkeiten innerhalb einer Phrase. Während die hierarchischen Strukturen mit Objektpermutationen eine Aktivierung im Broca-Areal evozierten (der linke inferior frontale Gyrus, insbesondere BA44/45), die je nach Anzahl der für die Rekonstruktion der Satzstruktur notwendigen Operationen variierte Röder et al. (2002); Ben-Shachar et al., (2003, 2004), involvierte die Verarbeitung von lokalen strukturellen Abhängigkeiten innerhalb einer Phrase (Friederici et al. 2003) und in adjazenten Phrasen (Ni et al. 2000; Opitz \& Friederici 
2004) das frontale Operculum. ${ }^{3}$ Die Untersuchung von Friederici et al. (2006) ergab eine Beteiligung des linken frontalen Operculum an der Verarbeitung sowohl der FSG- als auch PSG-Strukturen; das Broca-Areal (BA 44/45) war jedoch nur bei der Herstellung der rekursiv-hierarchischen Abhängigkeiten aktiv. Die Forscher haben daraus den Schluss gezogen, dass das linke frontale Operculum die Überprüfung ankommender Elemente einer Inputkette überwacht, womit zugleich das Engagement dieser Gehirnregion bei der Verarbeitung von lokalen Verletzungen erklärt werden kann. ${ }^{4}$ Demgegenüber wird das Broca-Areal (insbesondere der posterior inferiore Teil des Brodmann-Areals BA44, d.h. die inferiore Spitze von pars opercularis) bei der Verarbeitung von strukturellen Hierarchien zusätzlich einbezogen, wobei seine Aktivierung nicht mit Verarbeitungsschwierigkeiten per se, sondern mit der hierarchischen Struktur korreliert. Dies liefert somit eine Evidenz für die funktionelle Unterscheidung des Broca-Areals und des linken frontalen Operculum. ${ }^{5}$

\section{Domänenspezifität der neuronalen PSG-Verarbeitungsmechanismen}

In der Fachliteratur herrscht kein Konsens über die Domänenspezifität der funktionellen Mechanismen, die der Verarbeitung syntaktisch komplexer, hierarchischer Strukturen zugrunde liegen. Einige Forscher führen sie auf sprachinhärente Funktionen zurück, die mit der Anwendung komplexer linguistischer Operationen verbunden sind z.B. Embick et al. (2000); Grodzinsky (2000); Ben-Shachar et al. (2003, 2004). Andere assoziieren sie mit einer höheren Belastung des Arbeitsgedächtnisses Caplan et al. (2000); Fiebach, Schlesewsky \& Friederici (2001); Kaan \& Swaab, (2002). ${ }^{6}$ Der Grund für die große Meinungsverschiedenheit in dieser Hinsicht liegt darin, dass die Zunahme der Satzkomplexität mit der

${ }^{3}$ Das Areal im linken inferior frontalen Gyrus, das an den inferioren Teil des Broca-Areals angrenzt, aber medialer lokalisiert ist.

${ }^{4}$ Die Beteiligung dieser Region wurde z. B. beim Erscheinen eines durch die Grammatik nicht vorausgesagten lexikalischen Elements beobachtet Friederici, Rüschemeyer et al. (2003); Grewe et al. (2005). Dass das frontale Operculum sich an dem lokalen Phrasenstrukturaufbau beteiligt, bestätigt auch die Studie von Friederici, Meyer, \& von Cramon (2000).

${ }^{5}$ Das Broca-Areal und das linke frontale Operculum sind auch phylogenetisch distinkt. Das Broca-Areal stellt einen jüngeren Kortextteil dar als das linke frontale Operculum Brodmann (1909). Außerdem wird auch die zyto- und rezeptoarchitektonische Spezifität der beiden Areale betont Amunts et al. (1999); Zilles et al. (2002).

${ }^{6} \mathrm{Zu}$ beachten ist dabei, dass diejenigen Ansätze, die eine erhöhte Aktivierung im Broca-Areal auf die Belastung des Arbeitsgedächtnisses zurückführen, entweder zwei Arbeitsgedächtnissysteme, ein syntaktisches und ein nicht-syntaktisches Waters \& Caplan, (1992); Caplan \& Waters (1999); Fiebach, Schlesewsky \& Friederici (2001); Fiebach et al. (2005) oder nur ein domänenunspezifisches Arbeitsgedächtnis Just \& Carpenter (1992) postulieren. Die Kontroverse betrifft somit die Beteili- 
Erhöhung der Arbeitsgedächtniskosten einhergeht und es nicht auszuschließen ist, dass die erhöhte Aktivierung im Broca-Areal mit höheren Anforderungen an das Arbeitsgedächtnis verbunden ist. Es kann schwerfallen, die Evidenz für beide konkurrierende Ansätze voneinander zu trennen, weil die Arbeitsgedächtniskosten und syntaktische Komplexität in experimentellen Bedingungen oft zusammenfallen.

Die Studie von Friederici et al. (2006) eröffnete eine neue Perspektive für die Erforschung der Domänenspezifität des Broca-Areals. Insbesondere wurde eine neue Untersuchung durchgeführt Bahlmann et al. (2009) mit dem Ziel, die Aktivierung dieser Hirnregion bei der Verarbeitung der nicht-sprachlichen (visuellen) linearen und hierarchischen Sequenzen zu beobachten und somit auf die Domänen(un)spezifität des Broca-Areals zu schließen. Die Relation zwischen den abhängigen Elementen beim visuellen Input wurde durch Rotation definiert. Bahlmann et al. (2009) berichten über eine Aktivierung im präsupplementär-motorischen Kortex (SMA) und im rechten Nucleus caudatus beim visuell dargebotenen Input. Darüber hinaus wurde auch eine erhöhte hämodynamische Antwort im BA44 bei der Verarbeitung von rekursiv-hierarchischen Strukturen beobachtet, was darauf hinweist, dass das Broca-Areal seine Domänenspezifität als Teil eines bestimmten neuronalen Netzes empfängt. So unterstützt es zusammen mit dem posterior-superior temporalen Kortex die Verarbeitung der natürlichen Sprache z.B. Bornkessel et al. (2005), während dieselbe Hirnregion als Teil eines größeren neuronalen Netzes (mit SMA) in die Verarbeitung des nicht-sprachlichen visuellen Inputs involviert ist.

Die Dynamik der Verarbeitung von FSG- und PSG-Strukturen wurde in der früheren EKP-Studie von Bahlmann et al. (2006) erforscht. Den Input bildeten bedeutungslose Silbensequenzen, in denen zwei Kategorien (A und B) benutzt wurden und entweder einer lokal organisierten FSG-Regel oder einer hierarchisch organisierten PSG-Regel folgten. Die Untersuchung ergab eine Negativierung zwischen 300 und 400 ms für FSG und eine Positivierung zwischen 400 und 750 ms für beide Grammatiktypen. Die Befunde erklärten Bahlmann et al. (2006) damit, dass eine bewusste Erkennung eines spezifischen Items, das die Erwartungen des Parsers nicht erfüllt, durch die Negativierung reflektiert wird. Die Verarbeitung der lokalen Übergangswahrscheinlichkeit zwischen einzelnen Kategorien führt ihrer Meinung nach zur Erwartung einer spezifischen Kategorie, d.h., dass der Kategorie A immer die Kategorie B folgt. Falls eine andere Kategorie erscheint und diese Erwartung nicht erfüllt wird, wird eine Regelverletzung detektiert. Die Beobachtung, dass bei PSG-Verletzungen keine Negativierung hervorgerufen wurde, spricht für kompliziertere Mechanismen der PSG-Verarbeitung als bloßes Zusammenbringen der Übergangswahrscheinlichkeiten.

gung des sprachspezifischen und sprachunspezifischen Arbeitsgedächtnisses an der Verarbeitung syntaktisch komplexer hierarchischer Strukturen. 
Besondere Aufmerksamkeit gilt der Tatsache, dass die Amplitude der späten Positivierung in der Studie von Bahlmann et al. (2006) je nach Grammatiktyp und struktureller Verletzungsposition variierte. Frühe Verletzungen wurden in der ersten Sequenzhälfte an den Positionen 3 und 4 und späte Verletzungen in der zweiten Sequenzhälfte an den Positionen 6 und 7 angesiedelt. Für die FSG wurden keine Differenzen in der Amplitude in Abhängigkeit mit der Verletzungsposition gefunden. Im Gegensatz dazu evozierte PSG eine späte Positivierung mit einer größeren Amplitude für die späten und einer kleineren für die frühen Verletzungen. Dies ist damit zu erklären, dass in FSG die lokale Übergangswahrscheinlichkeit durch die Position nicht determiniert ist. In PSG ist jedoch die Verletzungsposition funktionell relevant und die Variation der Amplitude lässt sich nicht auf die Erwartung eines Items zurückführen.

Die genannten empirischen Befunde zur Verarbeitung der FSG- und PSG-Regeln verweisen zwar auf eine funktionelle Trennung der neuronalen Mechanismen ihrer Verarbeitung, doch lassen sie die Frage offen, ob die Effekte, die für die PSG-Verarbeitung spezifisch sind, d.h. die Aktivierung im linken pars opercularis und Variation der Amplitude je nach Verletzungsposition, ausschließlich durch die strukturelle Hierarchie erklärt werden und als Beleg für die Domänenspezifität der PSG-Verarbeitungsmechanismen gelten können. Insbesondere ist zu fragen, ob die beobachteten Effekte auf die syntaktische Komplexität allein oder auf die domänenunspezifischen Operationen des Arbeitsgedächtnisses zurückzuführen sind, dessen Ressourcen in die Verarbeitung der eingebetteten Strukturen einbezogen werden.

Einen Versuch, die Effekte der strukturellen Komplexität von Effekten der erhöhten Arbeitsgedächtniskosten bei der Verarbeitung von Zentraleinbettungen voneinander zu trennen, haben Makuuchi et al. (2009) unternommen. Die zentraleingebetteten Strukturen des Deutschen lieferten hinreichend Stoff, um die grundlegenden Eigenschaften der PSG-Verarbeitung zu untersuchen. Es wurden vier experimentelle Bedingungen konstruiert: mit und ohne Zentraleinbettung sowie mit höheren und niedrigeren Anforderungen an das Arbeitsgedächtnis, d.h. mit einem längeren und kürzeren Abstand zwischen syntaktisch abhängigen Konstituenten. Das Experiment deckte auf, dass die Prozesse der Syntaxverarbeitung und Arbeitsgedächtnisprozesse im linken inferior frontalen Gyrus separat operieren. Die Aktivierung im linken pars opercularis variierte als Funktion der Satzkomplexität, während Aktivierungen im linken inferior frontalen Sulcus (dorsal zum Broca-Areal gelegen) durch den Abstand moduliert wurden. Die Befunde bestätigen somit die These, dass die erhöhte Aktivierung in pars opercularis bei der Verarbeitung der zentraleingebetteten Strukturen durch den Abstand zwischen den abhängigen Elementen allein nicht erklärt werden kann, sondern dadurch, dass hier eine rekursiv-hierarchische Struktur mit einbezogen wird, die dem Strukturaufbau zugrunde liegt.

Die neuesten Untersuchungen mit der Diffusions-Tensor-Bildgebung (DTI) haben zwischen dem linken pars opercularis und dem linken inferior frontalen 
Sulcus eine anatomische Verbindung mit Fasern der weißen Substanz aufgedeckt Friederici et al. (2006); Makuuchi et al. (2009); auch Makuuchi \& Friederici (2013), was darauf schließen lässt, dass die beiden Hirnareale bei der Satzverarbeitung kooperieren. Bei der Verarbeitung zentraleingebetteter Strukturen können zusätzliche Arbeitsgedächtnisressourcen erforderlich sein. Diese zusätzlichen Arbeitsgedächtnisressourcen werden durch links inferior frontales Sulcus zur Verfügung gestellt, um mit partiellen Produkten des Parsingprozesses zurechtzukommen.

Die DTI-Daten sind auch aus der phylogenetischen Perspektive relevant. Das frontale Operculum, das der Verarbeitung der FSG-Strukturen zugrunde liegt, stellt ein phylogenetisch älteres Hirnareal dar als das Broca-Areal, das für die PSG-Verarbeitung verantwortlich ist Amunts \& Zilles (2012); Friederici (2014). $\mathrm{Zu}$ beachten ist auch, dass sich die Menschen und nicht-menschlichen Primaten in ihrem neuronalen Konnektivitätsmuster unterscheiden Rilling et al. (2008). Die Verbindung des Broca-Areals mit temporalen Hirnregionen über den Fasciculus arcuate, der eine Schlüsselrolle bei der Syntaxverarbeitung spielt, fällt bei Makaken aus und ist bei Schimpansen deutlich schwächer als bei Menschen. Da es jedoch keine Daten gibt, die einen direkten quantitativen und qualitativen Vergleich zwischen Menschen und nicht-menschlichen Primaten erlauben, befindet sich dieses Forschungsprogramm noch in der Anfangsphase.

\section{Fazit}

Die aktuelle Forschung zur Verarbeitung der rekursiv-hierarchischen und sequenziell-linearen Strukturen liefert den Beweis für eine funktionelle Trennung der neuronalen FSG- und PSG-Verarbeitungsmechanismen. Bei der Verarbeitung der rekursiv-hierarchischen Strukturen werden das Broca-Areal (pars opercularis) und der sequenziell-linearen das frontale Operculum aktiviert. Eine wichtige Entdeckung dabei ist, dass die PSG-Verarbeitungsmechanismen den nicht-menschlichen Primaten unzugänglich sind. Beachtenswerterweise sind beide Mechanismen in die Verarbeitung der natürlichen Sprache involviert. Mit den dargelegten Befunden ist aber keinesfalls das letzte Wort zur neuronalen Implementierung der Verarbeitung von rekursiv-hierarchischen Sprachstrukturen gesprochen. Trotz intensiver wissenschaftlicher Auseinandersetzung sind noch viele Fragen offen. Eine der grundlegendsten betrifft die Abgrenzung der strukturellen Komplexität von Arbeitsgedächtniskosten im experimentellen Design. $\mathrm{Zu}$ fragen ist, ob es überhaupt möglich ist, zwischen den Arbeitsgedächtnisprozessen und Prozessen der Verarbeitung von mentalen Repräsentationen eine scharfe Linie zu ziehen.

Mit der Entwicklung der modernen Techniken wie DTI-Bildgebung wurden für die neurolinguistische Forschung neue Wege eröffnet, was hoffentlich 
zu einem Erkenntnissprung führt. Zugleich hat sich auch die Perspektive auf die Frage nach der Lokalisation der sprachspezifischen Fähigkeiten verschoben. Die Domänenspezifität des Broca-Areals für die Syntaxverarbeitung scheint sich aus seiner Einbindung in ein spezifisches neuronales Netz zu ergeben. Mit dem posterior superior temporalen Kortex soll es die Verarbeitung der natürlichen Sprache und als Teil eines größeren neuronalen Netzes (mit SMA) die Verarbeitung des nicht-sprachlichen visuellen, hierarchisch organisierten Inputs unterstützen. Fraglich ist jedoch, ob die mit diesen Techniken gewonnenen Befunde, die klaffende Erkenntnislücke zwischen der unteren, zellulären und der oberen Organisation des Gehirns, die nur Reflex von Prozessen innerhalb kleinerer und größerer Zellverbände ist, schließen können.

\section{Literatur}

Amunts, K./ Schleicher, A./ Bürgel, U./ Mohlberg, H./ Uylings, H.B./ Zilles, K. (1999): Broca's region revisited: Cytoarchitecture and intersubject variability. In: The Journal of Comparative Neurology, 412(2), S. 319-341.

Amunts, K., Zilles, K. (2006): A Multimodal Analysis of Structure and Function in Broca's Region. In: Y. Grodzinsky/ K. Amunts (Hrsg.), Broca's Region. New York. S. 17-30.

Bahlmann, J./ Gunter, T.C./ Friederici, A.D. (2006): Hierarchical and Linear Sequence Processing: An Electrophysiological Exploration of Two Different Grammar Types. In: Journal of Cognitive Neuroscience, 18(11), S. 1829-1842.

Bahlmann, J./ Schubotz, R.I./ Mueller, J.L./ Koester, D./ Friederici, A.D. (2009): Neural circuits of hierarchical visuo-spatial sequence processing. In: Brain Research, 1298, S. 161-170.

Ben-Shachar, M./ Hendler, T./ Kahn, I./ Ben-Bashat, D./ Grodzinsky, Y. (2003): The neural reality of syntactic transformations. Evidence from fMRI. In: Psychological Science, 14, S. 433-440.

Ben-Shachar, M./ Palti, D./ Grodzinsky, Y. (2004): Neural correlates of syntactic movement: converging evidence from two fMRI experiments. NeuroImage, 21, S. 1320-1336.

Boeckx, C. (2010): Language in Cognition. Uncovering Mental Structures and the Rules Behind Them. Malden.

Bornkessel, I./ Zyssett, S./ Friederici, A.D./ von Cramon, D.Y./ Schlesewsky, M. (2005): Who did what to whom? The neural basis of argument hierarchies during language comprehension. In: NeuroImage, 26, S. 221-233.

Brodmann, K. (1909): Vergleichende Lokalisationslehre der Großhirnrinde in ihren Prinzipien dargestellt auf Grund des Zellenbaues. Leipzig.

Caplan, D./ Alpert, N./ Waters, G./ Olivieri, A. (2000): Activation of Broca's area by syntactic processing under conditions of concurrent articulation. In: Human Brain Mapping, 9, S. 65-71.

Caplan, D./ Waters, G.S. (1999): Verbal working memory and sentence comprehension. In: Behavioral and Brain Sciences, 22, S. 77-94.

Chomsky, N. (1956): Three models for the description of language. In: IRE Transactions on Information Theory IT-2, 113-124. Reprinted in Luce, Bush \& Galanter (1965), S. 105-124.

Chomsky, N. (1986): Knowledge of language: Its nature, origin, and use. New York.

Embick, D./ Marantz, A./ Miyashita, Y./ O’Neil, W./ Sakai, L. (2000): A syntactic specialization for Broca's area. In: Proc. Nati. Acad. Sci. USA, 97, S. 6150-6154.

Epstein, S./ Hornstein, N. (2005): Letter on 'The future of language'. In: Language, 81, S. 3-6. 
Everett, D.L. (1986): Pirahã. In D.C. Derbyshire/ G.K. Pullum (Hrsg.), The Handbook of Amazonian Languages, vol I. Berlin. S. 220-326.

Everett, D.L. (2005): Cultural constraints on grammar and cognition in Pirahã. In: Current Anthropology, 46(4), S. 621-646.

Everett, D.L. (2007a): Challenging Chomskyan Linguistics: The Case of Pirahã. In: Human Development, 50, S. 297-299.

Everett, D.L. (2007b): Cultural constraints on grammar in Pirahã: A reply to Nevins, Pesetsky and Rodrigues (2007). In: LingBuzz article archive: http://ling.auf.net/lingBuzz/000427.

Fiebach, C.J./ Schlesewsky, M./ Friederici, A.D. (2001): Syntactic working memory and the establishment of filler-gap dependencies: Insights from ERPs and fMRI. In: Journal of Psycholinguistic Research, 30 (3), S. 321-338.

Fiebach, C.J./ Schlesewsky, M./ Lohmann, G./ von Cramon, D.Y./ Friederici, A.D. (2005): Revisiting the role of Broca's area in sentence processing: Syntactic integration versus syntactic working memory. Human Brain Mapping, 24, S. 9-91.

Fitch, W.T./ Hauser, M.D. (2004): Computational constraints on syntactic processing in a nonhuman primate. Science, 303(5656), S. 377-380.

Fitch, W.T./ Hauser, M.D./ Chomsky, N. (2005): The evolution of the language faculty: clarifications and implications. Cognition, 97(2), S. 179-210.

Fitch, W.T./ Friederici, A.D. (2012): Artificial grammar learning meets formal language theory: an overview. Philosophical Transactions of the Royal Society B: Biological Sciences, 367, S. 1933-1955.

Friederici, A.D. (2014): Is There a Brain Basis of Recursion? In: F. Lowenthal/ L. Lefebvre (Hrsg.), Language and Recursion. New York u.a. S. 101-114.

Friederici, A.D./ Bahlmann, J./ Heim, S./ Schubotz, R.I./ Anwander, A. (2006). The brain differentiates human and non-human grammars: Functional localization and structural connectivity. In: Proceedings of the National Academy of Sciences USA 103, S. 2458-2463.

Friederici, A.D., Meyer, M., von Cramon, D.Y. (2000): Auditory Language Comprehension: An Event-Related fMRI Study on the Processing of Syntactic and Lexical Information. In: Brain and Language, 75(3), S. 465-477.

Friederici, A.D./ Rüschemeyer, S.-A./ Hahne, A./ Fiebach, C.J. (2003): The role of left inferior frontal and superior temporal cortex in sentence comprehension: Localizing syntactic and semantic processes. In: Cerebral Cortex, 13, S. 170-177.

Grewe, T./ Bornkessel, I./ Zysset, S. (2005): The emergence of the unmarked: A new perspective on the language-specific function of Broca's area. In: Human Brain Mapping, 26, S. 178-190.

Grodzinsky, Y. (2000): The neurology of syntax: Language use without Broca's area. Behavioral and Brain Sciences, 23, S. 1-71.

Hauser, M.D./ Chomsky, N./ Fitch, W.T. (2002): The faculty of language: what is it, who has it, and how did it evolve? In: Science, 298(5598), S. 1569-1579.

Jackendoff, R./ Pinker, S. (2005): The nature of the language faculty and its implications for evolution of language (reply to Fitch, Hauser, and Chomsky). In: Cognition, 97(2), S. 211-225.

Just, M.A./ Carpenter, P.A. (1992): A capacity theory of comprehension: Individual differences in working memory. In: Psychological Review, 99, S. 122-149.

Kaan, E./ Swaab, T.Y. (2002): The brain circuitry of syntactic comprehension. In: Trends in Cognitive Sciences, 6, S. 350-356.

Makuuchi, M./ Bahlmann, J./ Anwander, A./ Friederici, A.D. (2009): Segregating the core computational faculty of human language from working memory. In: Proc. Natl Acad. Sci. USA, 106, S. 8362-8367.

Makuuchi, M./ Friederici, A.D. (2013): Hierarchical functional connectivity between the core language system and the working memory system. In: Cortex, 49(9), S. 2416-2423. 
Marcus, G. (2006): Startling starlings. In: Nature, 440, S. 1204-1207.

Martins, M.D./ Fitch, W.T. (2014): Investigating Recursion Within a Domain- Specific Framework. In: F. Lowenthal/ L. Lefebvre (Hrsg.), Language and Recursion. New York u.a. S. 15-26.

Moro, A. (2008): The Boundaries of Babel. The Brain and the Enigma of Impossible Languages. Cambridge, MA.

Nevins, A.I./ Pesetsky, D./ Rodrigues, C. (2009): Pirahã exceptionality: A reassessment. In: Language, 85(2), S. 355-404.

Ni, W./ Constable, R.T./ Mencl, W.E./ Pugh, K.R./ Fulbright, R.K./ Shaywith, B.A./ Gore, J.C./ Shankweiler, D. (2000): An Event-related Neuroimaging Study Distinguishing Form and Content in Sentence Processing. In: Journal of Cognitive Neuroscience, 12, S. 120-133.

Opitz, B./ Friederici, A.D. (2004): Brain correlates of language learning: the neuronal dissociation of rule-based versus similarity-based learning. In: Journal of Neuroscience, 24(39), S. 84368440.

Pinker, S./ Jackendoff, R. (2005): The faculty of language: what's special about it?. Cognition, 95(2), S. 201-236.

Pullum, G.K/ Scholz, B.C. (2010): Recursion and the infinitude claim. In: H. van der Hulst (Hrsg.), Recursion and human language. Berlin, New York. S. 113-138.

Rilling, J.K./ Glasser, M.F./ Preuss, T.M./ Ma, X.Y./ Zhao, T.J./ Hu, X.P./ Behrens, T.E.J. (2008): The evolution of the arcuate fasciculus revealed with comparative DTI. In: Nature Neuroscience, 11(4), S. 426-248.

Röder, B./ Stock, O./ Neville, H./ Bien, S./ Rösler, F. (2002): Brain activation modulated by the comprehension of normal and pseudo-word sentences of different processing demands: A functional magnetic resonance imaging study. In: NeuroImage, 15, S. 1003-1014.

Sakel, J. (2007): On Pirahã and Everett's claims about recursion. In: Human Development, letters to the editor, 50(6).

Sakel, J./ Stapert, E. (2010). Pirahã - in need of recursive syntax?. In: H. van der Hulst (Hrsg.), Recursion and human language. Berlin, New York. S. 3-16.

Slobin, D. (2007): Context and comments on Dan Everett's claims. In: Human Development, letters to the editor, 50(6).

Yang, Ch. (2006): The Infinite Gift. New York.

Waters, G.S./ Caplan, D. (1992): The capacity theory of sentence comprehension: critique of Just and Carpenter (1996). In: Psychological Review, 103, S. 761-772.

Zilles, K./ Schleicher, A./ Palomero-Gallagher, N./ Amunts, K. (2002): Quantitative analysis of cytoand receptor architecture of the human brain. In: J.C. Mazziotta, A. Toga, S. 573-602.

Zwart, J.-W. (2011): Recursion in Language: A Layered-Derivation Approach. In: Biolinguistics, 5(1-2), S. 043-056.

\section{Abstracts}

Im vorliegenden Aufsatz wird die Frage nach der potentiellen Implementierung der rekursiven $\mathrm{A}^{\mathrm{n}} \mathrm{B}^{\mathrm{n}}-$ -Strukturen durch den Mechanismus der Satzverarbeitung behandelt. Es wird darauf hingewiesen, dass die einfachste Verarbeitungsstrategie, wo die adjazenten Sequenzen von zwei Kategorien A und B aufgezählt und verglichen werden, wegen der asymmetrischen Struktur der zentral-eingebetteten syntaktischen Abhängigkeiten keine Anwendung bei der kognitiven Verarbeitung finden kann.

Schlüsselwörter: Rekursion, syntaktische Komplexität, Satzverarbeitung, Satzverstehen 


\title{
Recursion and syntactic complexity: Psycholinguistic and neurolinguistic perspectives
}

This article is devoted to the issue of the potential implementation of recursive $\mathrm{A}^{\mathrm{n}} \mathrm{B}^{\mathrm{n}}$ structures by the sentence-processing mechanism. Worth noting is the fact that the simplest processing strategy, whereby categories A and B are listed and compared, cannot be applied to the cognitive processing of asymmetric structures of centrally embedded syntactic dependencies.

Keywords: recursion, syntactic complexity, sentence processing, sentence comprehension

\author{
Jolanta Sękowska \\ Uniwersytet Marii Curie-Skłodowskiej, Lublin \\ Wydział Humanistyczny \\ Instytut Germanistyki i Lingwistyki Stosowanej \\ Pl. Marii Curie-Skłodowskiej 4a \\ 20-031 Lublin \\ Polen \\ E-Mail: jolanta.sekowska@poczta.umcs.lublin.pl
}

